\title{
Role of Antigravity Training in Rehabilitation and Return to Sport After Running Injuries
}

\author{
Heather K. Vincent, M.D., Ph.D., Aimee Madsen, M.D., and Kevin R. Vincent, M.D., Ph.D.
}

\begin{abstract}
Anti-gravity treadmill training is a therapeutic option to help recovering runners return to activity after injury. This current concept paper provides a synopsis of the latest evidence of the biomechanical and metabolic changes that occur with body weight support (BWS) treadmill training, effects of antigravity treadmill training on clinical outcomes and clinical case studies in injured runners. Literature searches identified studies with descriptive, experimental and interventional designs and case studies that examined acute and chronic use of antigravity treadmills in runners and relevant populations. Laboratory-based studies were included to provide technical considerations for rehabilitation programming. Antigravity treadmills use causes reductions in cadence, ground reaction forces (GRF), GRF impulses, knee and ankle range of motion, and vertical stiffness, with elevations in stride duration, flight time, ground contact time, and plantarflexion. Antigravity treadmills appear useful across a spectrum of injuries in runners, including postsurgical repair of osteochondral defect, stress reactions (medial tibia, pelvis), and lumbar disc herniation. Runners may preserve aerobic fitness, muscle activation patterns, and muscle mass during recovery compared to traditional rehabilitation protocols. Technical considerations for accurate loading include treadmill frame adjustment to appropriate height to ensure accuracy of level of BWS while running, and monitoring for fast cadence to ensure impact loading rates remain low. Speed or grade can be increased to maintain metabolic demand and fitness while minimizing bone and tissue loading. Monitoring for symptom provocation will guide protocol adjustments to BWS and prescriptions. Once able to run pain-free (sustained or interval) $>95 \%$ BWS for $>30 \mathrm{~min}$, the runner is likely ready to safely transition to ground running. Antigravity treadmill training can be considered when available to facilitate smooth transition back to ground running in a conditioned state.
\end{abstract}

\section{Introduction}

$\mathbf{R}$ ehabilitation from running-related injury often involves cross-training activities with relatively little or no physical impact to joints to allow the musculoskeletal system to heal without losing cardiorespiratory fitness. These activities can include elliptical

Department of Physical Medicine and Rehabilitation, University of Florida College of Medicine, Gainesville, Florida, 32611, U.S.A.

The authors report the following potential conflicts of interest or sources of funding: Drs H.K.V. and K.R.V. are both Associate Editors for Medicine and Science in Sports and Exercise. Dr. Heather Vincent's research is supported, in part, by Lallemand, Inc. Full ICMJE author disclosure forms are available for this article online, as supplementary material.

Received September 2, 2021; revised manuscript received September 21, 2021; accepted September 22, 2021.

Address correspondence to Heather K. Vincent, PhD, FACSM, Department of Physical Medicine $\theta$ Rehabilitation, UF Health Sports Performance Center, 3450 Hull Rd., P.O. Box 112730, University of Florida, Gainesville, FL, 32611,U.S.A.E-mail: hkvincent@ufl.edu

(C) 2021 THE AUTHORS. Published by Elsevier Inc. on behalf of the Arthroscopy Association of North America. This is an open access article under the CC BY-NC-ND license (http://creativecommons.org/licenses/by-nc-nd/4.0/). 2666-061X/211303

https://doi.org/10.1016/j.asmr.2021.09.031 training or pool training (deep water running). ${ }^{1}$ Over recent years, more scientific evidence is emerging to demonstrate value of antigravity training for running rehabilitation. Antigravity treadmills use positive air pressure to provide partial body-weight support (BWS), thereby lowering impact forces and metabolic demand of running. In this current concept article, potential benefits of antigravity treadmill training and expected biomechanical and cardiometabolic responses are presented, with technical considerations in program development. Available intervention studies and case reports using BWS treadmills are shared, with sample antigravity treadmill protocols specific to rehabilitation in runners.

Literature searches were conducted in several databases (CINAHL, PubMed, MEDLINE Ovid, Scopus) from 1980 to the present to capture relevant experimental, interventional, and clinical evidence of antigravity treadmill's effects on running biomechanics and physiological responses and on clinical outcomes, as well as considerations when transitioning from antigravity treadmill training back to overground running. 


\section{Support Mechanism of the Antigravity Treadmill}

Antigravity treadmills leverage ideas from wellestablished technologies, such as body weight harness systems and underwater treadmills. Although these systems support body weight directly with cables or provide body buoyancy in water, the anti-gravity treadmill uses positive pressure from compressed air in an enclosed air-tight structure. ${ }^{2}$ The enclosed system surrounds the lower half of the participant's body. A treadmill is surrounded over the top by an air-tight enclosed inflatable bag, and the participant wears a special set of shorts that have half a zipper around the hip circumference that then zip into the top of the inflatable bag. ${ }^{3}$ The inflatable bag is attached at each side to support poles that are parallel to the lateral sides of the zipper and are vertically adjustable set into the outer frame of the antigravity treadmill. Vertical adjustment of the frame height allows participants of different heights to use the machine. ${ }^{4}$ Moreover, a more sensitive adjustment of the body weight support can be provided. Antigravity treadmills allow for support up to $80 \%$ of body weight. ${ }^{2}$

\section{Antigravity Treadmill Training for Rehabilitation of Running Injuries}

The benefits of antigravity treadmills in rehabilitation include early mobilization with similar motion stimuli as ground running without the harmful impact on injured tissues. Importantly, athletes can maintain cardiopulmonary fitness through manipulation of treadmill speed or grade until resumption of full loading with ground running.

\section{Early Mobilization and Strength Preservation}

With BWS, walking and running can be incorporated earlier in the process of recovery when full-weight bearing activities are often not clinically recommended or tolerated by the individual. Immobilization from surgery and non-weight bearing phases of rehabilitation contribute to loss of leg muscle mass and strength and delayed return to running. Use of antigravity treadmills may offset these effects and prevent muscle atrophy and weakness while minimizing discomfort. Even among persons with hip fracture injury, early treadmill use during weeks 1-4 postinjury hip extension strength and gluteal muscle activities were better preserved than with standard rehabilitation alone. ${ }^{5}$ For runners, preservation of strength and muscle activation patterns could speed the return to ground running.

\section{Maintenance of Physical Conditioning}

Preservation of cardiorespiratory fitness during recovery will facilitate a seamless return to running, especially for competitive endurance athletes, as a $7 \%$ decline in peak rate of oxygen consumption $\left(\mathrm{VO}_{2}\right)$ occurs within 2-3 weeks of training cessation. ${ }^{6}$ While a rehabilitated runner may return to the sport healthy, the athlete may be undertrained. ${ }^{7}$ Antigravity treadmills offer variations in speed and elevation, while protecting the runner from bone and soft tissue impact. Unloaded running can allow an athlete to run at faster intensities earlier in the rehabilitation and recovery process compared to overground running without the bone or tissue loading. ${ }^{8}$ Previous studies have shown that increasing speed with BWS can produce submaximal and maximal metabolic demands comparable to those of overground running. ${ }^{9-12}$ Similarly, when treadmill incline is increased from $1 \%$ to $15 \%$ at a given speed, $\mathrm{VO}_{2}$ increases proportionately; at an incline of $7 \%$, the metabolic demand is equal to that of running with full body weight. (BW). ${ }^{7}$

\section{Expected Motion Changes with Antigravity Treadmill Use}

When a runner initiates rehabilitation on an antigravity treadmill, there are several expected changes to running motion that need to be considered. Temporalspatial, kinematic, and kinetic changes occur with varying levels of BWS, as described next. A summary of these motion changes is shown in Figure 1.

\section{Temporal-Spatial Parameters}

Among healthy runners, natural cadence will lessen with any level of BWS. ${ }^{13}$ When exposed to progressively higher BWS from $20 \%$ to $100 \%$, the cadence decreases from $1.5 \%$ to $3.5 \%$ per $10 \%$ unit of support away from the natural training cadence. ${ }^{13}$ Other investigators reported similar findings. ${ }^{14-17}$ Therefore, during rehabilitation programs, it is important to monitor cadence and adjust as needed through metronome targets to maintain a healthy cadence, as the runner transitions back to overground running. Stockland et al. found that across the spectrum of BWS levels, runners could achieve cadence targets 5\%-10\% higher than natural cadence. Commensurate with cadence, the vertical displacement of the center of mass during the braking phase of the gait cycle and push-off impulse are progressively reduced with additional treadmill support. ${ }^{16}$ This type of treadmill facilitates rehabilitation without the same mechanical stresses as a regular treadmill.

Running unweighted at $85 \%$ BW can increase stride length as much as $12 \%$ from normal, full-weighted running conditions. ${ }^{12}$ Stride length increases progressively with BWS. This effect should be monitored, as training over time (eight weeks, 3 times/week for 20 min per session, at $85 \% \mathrm{BW}$ ) may inadvertently lead to permanent and moderate $4.3 \%$ increases in stride length. ${ }^{18}$ Stride duration increases significantly with progressively more body weight support across several 
running speeds; most of this finding is attributed to extended flight time and less ground contact time. ${ }^{15,16}$

\section{Kinematics}

Analysis of ankle kinematics revealed increased plantar flexion at initial contact, reduced peak dorsiflexion, and reduced overall ankle range of motion, as unweighting was increased. ${ }^{19}$ The ankle is placed in a position of greater plantar flexion at initial contact and does not return to full dorsiflexion during stance. ${ }^{15}$ More BWS encourages more plantarflexion. Conflicting data exist for knee flexion/extension range of motion and peak knee flexion. ${ }^{15,19}$ One report did not find that knee range of motion changed with more BWS during running. ${ }^{19}$ Others report a reduction in peak knee flexion up to $33 \%$ when treadmill support increased from $0 \%$ to $80 \%$ body weight. ${ }^{15,20}$ Importantly, these relationships between ankle and knee motion relative to body weight do not change by running speed. ${ }^{15}$

\section{Kinetics, Vertical Stiffness, and Plantar Loading}

Experimental studies and systematic reviews consistently reveal that ground reaction force (GRF), impulses, and peak knee joint moments proportionally decrease with more BWS. ${ }^{1,14,16,17,19,21-23}$ Across a range of speeds, the sequential addition of BWS from $0 \%$ to $80 \%$ body weight reduces peak GRF, irrespective of foot strike type. ${ }^{15}$ An important finding by Moran et al. ${ }^{14}$ is that tibial acceleration and tibial shock magnitudes are not mitigated by unweighting if the cadence is simultaneously reduced. Rehabilitation teams should monitor cadence periodically to ensure that cadence is remaining in the range necessary to keep impact loading low especially in early phases of rehabilitation. In other studies, 1) plantar pressures shift toward a more forefoot loading pattern with incremental increases in support, ${ }^{23}$ and 2) vertical stiffness is also significantly reduced with progressive BWS. ${ }^{16}$ A clinically important finding of Smoliga et al. ${ }^{23}$ is that running mechanics can be largely retained with $20 \%$ body weight offloading, while GRF, impulses, and plantar loading are significantly reduced. Thus, with appropriately chosen cases, runners may retain running form while reducing mechanical loading at this $20 \%$ level. $^{23}$

\section{Muscle Activity Patterns}

Changes in muscle activity with different levels of unweighting are complex and not consistent across different muscle groups. ${ }^{16,17,24,25}$ Earlier studies demonstrated general reduction in muscle activity of the bicep femoris, rectus femoris, tibialis anterior, and gastrocnemius in healthy runners with progressive unloading and greater muscle activity at speeds at 115 $125 \%$ of preferred running speed at all levels of unweighting. ${ }^{25}$ Surface electromyography of lower extremity muscles (gastrocnemius [medialis and lateralis], tibialis anterior and vastus medialis, lateralis) collected during running with BWS shows that preactivation levels of these muscles do not change with unweighting. ${ }^{16}$ The authors attributed this finding to maintained muscle preactivation to counteract an anticipated impact from a longer flight time. ${ }^{16}$ Other investigators have demonstrated almost linear inverse reductions in rectus femoris, vastus medialis-lateralis, gastrocnemius, and soleus muscle activity with $20 \%$ increments in BWS. ${ }^{17,20}$ However, during braking and push-off, muscle activity of the soleus and gastrocnemius (medial and lateral) decreased by $10-20 \%$, with progressive BWS. ${ }^{16}$ Increasing the cadence by $10 \%$ from the habitual preferred value increases the muscle activity of the rectus femoris, biceps femoris, and tibialis anterior, irrespective of the amount of BWS. ${ }^{20}$ To maintain optimal muscle coordination during rehabilitation, cadence manipulation may be effective in preserving more natural muscle activation patterns with unweighting.

\section{Interventions with Anti-Gravity Treadmills}

The utility of antigravity treadmills has been shown even after severe orthopedic injury, such as ankle and tibial plateau fractures, ${ }^{26}$ Achilles tendon rupture, or insertional injury, ${ }^{2}$ or anterior cruciate ligament (ACL) injury. ${ }^{24}$ Case studies for runners with pelvis stress injury $^{27}$ and lumbar disc herniation ${ }^{28}$ support the use of unweighted running as part of the rehabilitation process. Following ankle fracture or tibial plateau fracture, common recovery and rehabilitation involves nonweight or partial weight bearing for weeks after the surgery to protect the joint, surgical implants and sutures, or soft tissue that has been reconstructed. Postoperatively, immobilized limbs develop muscle atrophy and strength loss, and joints stiffen. When the athlete returns to activity, return to preinjury training volume or performance will be prolonged until the muscle function is restored and reconditions to run training. While return-to-activity as soon as possible is important for athletes, the postsurgical considerations and postulated risk for reinjury make the rehabilitation process more challenging.

Henkelmann et al. ${ }^{26}$ examined whether these BWS treadmills would provide better postoperative rehabilitation outcomes than standard crutch use and partial weight-bearing protocols for isolated closed tibial or ankle fractures with partial weight-bearing status. Patients were randomized to either standard physiotherapy (with mobilization under partial weight bearing with crutches) or antigravity treadmill sessions with loading of $20 \mathrm{~kg}$ at $1 \%$ incline 2-3 times per week for 6 weeks. The treadmill speed was initially set at $1.5-2 \mathrm{~km} /$ $\mathrm{h}$ in a ratio of 5:5-minute bouts at each speed during the first 2 weeks. At week 6, the speed was increased to 4-5 $\mathrm{km} / \mathrm{h}$ in a ratio of 8:2-minute bouts. The most 
relevant finding was a better improvement in leg circumference over the 6-week period with antigravity treadmill training, accompanied by improvement in Knee Injury and Outcomes scores for function/sports and quality of life.

Patients who underwent a surgical procedure for Achilles tendon rupture or insertional repair were prospectively studied while undergoing standard rehabilitation alone or with antigravity treadmill training. ${ }^{2}$ Patients were kept non-weight bearing for 2 and 4 weeks, respectively. Patients with Achilles rupture progressed to initial weight bearing with a removable boot from 2 to 6 weeks and into athletic shoes with heel wedges at week 8. After Achilles tendon insertion repair, patients were kept in a below-the-knee boot or cast until week 4 , at which time, weight-bearing began. By week 10, patients progressed to an athletic shoe with a wedge. Both surgical groups followed a validated rehabilitation protocol with icing, therapeutic mobilization, strengthening, balance and modalities. At week four, antigravity treadmill training at $70 \%$ BWS was initiated with distances up to 2 miles. At week 5, BWS on the treadmill was decreased, and training continued at $75 \%-85 \%$ BWS for 2 miles. At week 6, a walk/run program was initiated (alternating between 2 minutes walking, 2 minutes running for 10 minutes). BWS ranged from $75 \%-85 \%$. When patients could successfully run at $85 \% \mathrm{BW}$, they were discharged to begin a return to run program outside. Patients who used the antigravity treadmill returned to running an average of 2 weeks faster than patients with traditional rehabilitation. The authors indicated value of this treadmill training from the standpoint of proper determination of readiness and clearance to run outside. Thus, antigravity treadmills offer individuals even with very severe injuries the opportunity to maintain leg tissue mass, maintain better quality of life, and to demonstrate readiness to return to running.

Patients with ACL reconstruction may undergo either bone-patellar tendon-bone or hamstring autograft procedures. Early reengagement into running after surgery can help mitigate the adverse effects of deconditioning during recovery. However, there has been concern that antigravity treadmills may foster deleterious motor patterns that could stress the lower extremity muscles or surgical site and increase the risk for reinjury. Hansen et al. ${ }^{24}$ performed a comparative experimental study of the acute muscle activation effects of antigravity treadmill running in patients rehabilitating from either of the two ACL reconstruction techniques indicated above relative to healthy controls. Electromyogram (EMG) activity of the plantar flexors soleus medialis, soleus lateralis, medial gastrocnemius, lateral gastrocnemius, and the medial/ lateral hamstrings were collected while athletes ran up to $16 \mathrm{~km} / \mathrm{h}$ with $50 \%-100 \%$ BWS in $10 \%$ increments. Healthy controls demonstrated reductions in EMG activity across all muscles with incremental unloading and more activity with increased running speed. Muscle activity did not differ between healthy controls and bone-patellar tendon-bone across all unweighting levels. Activity was different for the hamstring autograft versus controls only for the $90 \%-100 \%$ BWS conditions. ${ }^{24}$ Runners with ACL reconstruction may benefit from antigravity training without elevated risk for reinjury-but with additional BWS for patients with hamstring autograft.

A case report indicates successful rehabilitation for elite runners $(110+\mathrm{km} / \mathrm{wk})$ with lumbar disc herniation. ${ }^{28} \mathrm{~A}$ 52-year old male experienced acute back injury, progressive pain in all body positions, loss of sensation in right anterior lower leg, and diminished strength reflexes over the week after injury. Antigravity treadmill walking was initiated 6 days postinjury for $2.4 \mathrm{~km}$, with $50 \%$ body weight support. With no symptom worsening, the athlete walked $11 \mathrm{~km}$ at $4.5 \mathrm{~m} / \mathrm{s}$ with $50 \%$ BWS. The following week, training progressed to running with the same support at 5\% to reduce impact loading. During weeks 2 and 5, the athlete increased running speeds to reach heart rates above $150 \mathrm{bpm}$ with BWS of $70 \%-80 \%$. Weekly running distances ranged from $113 \mathrm{~K}$ to $153 \mathrm{~K}$, with one-fifth of the volume at $85 \%-90 \%$ BWS. Trail run simulations were introduced. After 6 weeks, the runner resumed regular training schedule of $>125 \mathrm{~km}$ per week. Symptoms resolved during recovery and strengthening exercises were progressively added. In the case of acute spine injury, antigravity treadmill training helped mitigate a cessation or precipitous drop in training volume and facilitated a rapid transition back to overground running.

While stress injuries are common among endurance runners, iliac stress injuries are not. Typically, 8-12 weeks of rest and modified activity are required for safe and appropriate bone remodeling. During this time, significant detraining and fitness decline occurs, which can interfere with performance during the competitive part of a season. Tenforde et al. ${ }^{27}$ described the treatment of a 21-year old female runner (with history of oligomenorrhea, osteopenia and prior metatarsal stress fractures) for persistent left buttock pain over 4 weeks. Magnetic resonance imaging of the pelvis revealed a stress reaction at the left ileum adjacent to the sacroiliac joint. Impact loading was initially restricted. Five days after diagnosis, the runner began isometric core and hips stabilization exercise and continued through week 2. At week 3, she attempted three runs with 50\%-70\% $\mathrm{BW}$, but this running provoked symptoms, and running was stopped completely until week 5 . Five weeks after diagnosis, she ran at 50\% BW (5 minutes of jogging and 1 minute recovery for 3 repetitions) with no pain. She was permitted to run every other day, increasing her running time by 5-15 minutes and decreasing BWS by 
$5-10 \%$ per run. During week 6 , she ran at $70 \%$ BW for 25 minutes and during week 7, she could run continuously for 35 minutes at $85 \%$ BW. At week 8 , she ran for 45 minutes at $95 \% \mathrm{BW}$, and she was then cleared for ground running. At week 10, she competed in the conference championship and qualified for the NCAA Track Championships at week 11 . She did not experience recurrence of pain or injury. The authors indicated the value of pain-free unweighted running to predict success with safe return to overground training.

\section{Technical Considerations for Accurate Unloading}

From the technical standpoint, the setup of the antigravity treadmill will affect the support level provided to the runner. For the AlterG treadmill model, a metal bar frame structure supports the sealed air bag over the treadmill. The frame position can be adjusted vertically to accommodate different body heights. The accuracy of unweighting may be impacted by the frame placement. The standard operating instructions of the treadmill states the frame should position the zipper at the iliac crest line, with the caveat that the frame can be adjusted up or down on the basis of the individual preference for more or less trunk stabilization. de Heer et al. ${ }^{4}$ examined the accuracy of the AlterG unweighting across a range of body weight support levels (from $0 \%$ to $60 \%$ ). Following the standard instructions, the authors found that the greatest accuracy occurred in the $10 \%-40 \%$ BWS range. Even at the displayed 0\% BWS, the treadmill still actually provided 6\% BWS. Moving the frame up or down from the level of the iliac crest produced significant changes to the accuracy of the unweighting. When the frame was set below the iliac crest, the treadmill provided 5\%-24\% less support than indicated for each level. When the frame was set above the iliac crest level, the support was considerably higher than indicated at each level; even at $0 \%$ support, an elevated frame provided $\sim 8 \%$ body weight support. The greatest deviations in accuracy occurred at $>40 \%$ BWS. Thus, moving the frame up or down to accommodate "patient preference" for truncal stability can unintentionally change the mechanical loading. These findings have important implications for the runner in the early stages of rehabilitation from injury, when the higher body weight support is used.

\section{Considerations When Transitioning from Antigravity Training Back to Ground Running}

\section{Accuracy of the Treadmill Support Level}

The accuracy of the amount of BWS provided by the treadmill varies along the support spectrum. Even with $0 \%$ BWS, the treadmill may, in reality, provide 5\%-8\% BWS. $^{4,29}$ An experimental study was conducted specifically to test the accuracy of the BWS proportions displayed by the AlterG treadmill. ${ }^{3}$ It was found that greatest variability from the predicted support level occurred at $100 \%$ (93\% actual BWS), and above $60 \%$ (actual BWS level was 3\%-8\% more than displayed). An important note is that these variations in accuracy are likely tolerable for individuals after surgery. It has been reported that these variations are less than those
Fig 1. Key temporal-spatial, kinematic, muscle activation, kinetic, and cardiometabolic responses to progressive body weight support on an antigravity treadmill. $\mathrm{BF}$, biceps femoris; COG, center of gravity; GAS, gastrocnemius; GRF, ground reaction force; $\mathrm{RF}$, rectus femoris; $\mathrm{TA}$, tibialis anterior; VL, vastus lateralis; SOL, soleus; VE, minute ventilation; $\mathrm{VM}$, vastus medialis; $\mathrm{VO}_{2}$, rate of oxygen consumption.

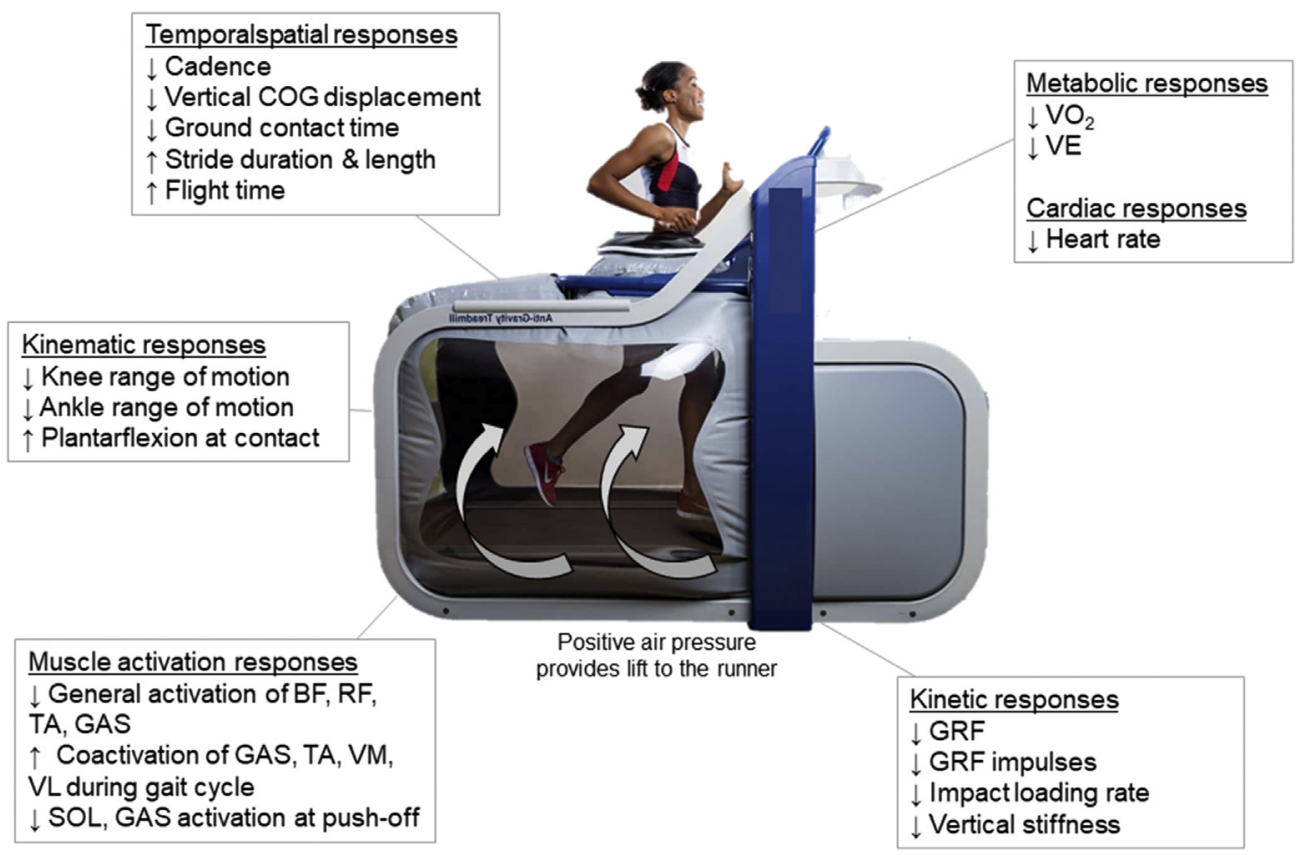




\author{
Recreational runner \\ Commonlyruns at $6 \mathrm{mph}(10: 00 \mathrm{~min} / \mathrm{mile})$
}

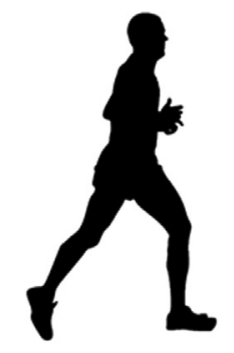

To match $\mathrm{VO}_{2}$ at with ground running:

$\begin{array}{ll}\begin{array}{l}\text { Treadmill } \\ \text { support }\end{array} & \text { Corresponding speed } \\ 50 \% \text { BWS } & 9.93 \mathrm{mph}(6: 02 \mathrm{~min} / \mathrm{mile}) \\ 60 \% \text { BWS } & 9: 33 \mathrm{mph}(6: 26 \mathrm{~min} / \mathrm{mile}) \\ 70 \% \text { BWS } & 8: 69 \mathrm{mph}(6: 54 \mathrm{~min} / \mathrm{mile}) \\ 80 \% \text { BWS } & 7: 89 \mathrm{mph}(7: 36 \mathrm{~min} / \mathrm{mile}) \\ 90 \% \text { BWS } & 7: 12 \mathrm{mph}(8: 26 \mathrm{~min} / \mathrm{mile}) \\ 100 \% \text { BWS } & 6: 50 \mathrm{mph}(9: 16 \mathrm{~min} / \mathrm{mile})\end{array}$

\author{
Elite marathon runner \\ Commonlyruns at $9.5 \mathrm{mph}(6: 19 \mathrm{~min} / \mathrm{mile})$
}

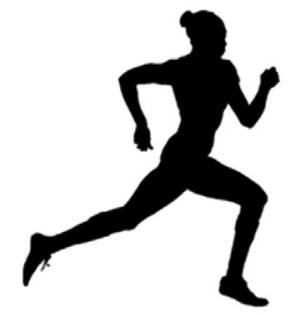

To match $\mathrm{VO}_{2}$ with ground running:

$\begin{array}{ll}\begin{array}{l}\text { Treadmill } \\ \text { support }\end{array} & \text { Corresponding speed } \\ 50 \% \text { BWS } & 14.34 \mathrm{mph}(4: 11 \mathrm{~min} / \mathrm{mile}) \\ 60 \% \text { BWS } & 13: 35 \mathrm{mph}(4: 30 \mathrm{~min} / \mathrm{mile}) \\ 70 \% \text { BWS } & 13: 08 \mathrm{mph}(4: 35 \mathrm{~min} / \mathrm{mile}) \\ 80 \% \text { BWS } & 11: 12 \mathrm{mph}(5: 24 \mathrm{~min} / \mathrm{mile}) \\ 90 \% \text { BWS } & 10: 02 \mathrm{mph}(5: 59 \mathrm{~min} / \mathrm{mile}) \\ 100 \% \text { BWS } & 9.63 \mathrm{mph}(6: 14 \mathrm{~min} / \mathrm{mile})\end{array}$

Fig 2. Sample speed conversions when using an antigravity treadmill to maintain aerobic fitness at varying body weight support (BWS) levels. Recreational and elite runner examples are provided.

Guided by Kline et al. (2015) J Strength Cond. 29(3):854-862.

observed with partial weight-bearing with crutches after cartilage repair of the knee. ${ }^{30}$ However, close symptom monitoring should occur as runners transition from earlier phases of rehabilitation with more body support to later stages with less BWS to prevent pain provocation and rehabilitation setbacks.

\section{Increase Running or Grade Speed to Preserve Fitness}

Unweighting running impacts the metabolic cost and cardiorespiratory parameters. $^{31,32}$ First, $\mathrm{VO}_{2}$ and ventilation decrease as BWS increases, ${ }^{3,11}$ but this decrease is nonlinear. ${ }^{3}$ This finding may be due to the progressive reduction in frontal/lateral stabilization provided by the treadmill ${ }^{20}$ and less metabolic demand from muscle stabilizers with more support. During the pain-free phases of rehabilitation, the speed of the treadmill could be increased while maintaining $85 \%$ to $90 \% \mathrm{BW}^{12}$ to achieve comparable $\mathrm{VO}_{2}$ experienced in the real training environment. Heart rates are progressively reduced for each increment in body weight support. Compared to full-weighted conditions, acute supported running decreased HR by $8.6 \%$ with $20 \%$ support, and $13.3 \%$ with $40 \%$ BWS. ${ }^{14}$ Similarly, other investigators have consistently found HR reduction with unweighting. $3,10,12,16,31$

As part of the readaptation back to ground running, a period of supported training at higher-than-normal speeds with partial body weight support may help expose the runner to the accustomed physiological training intensities before reintroducing full body weight. Alternatively, if speed is not appropriate for the injury being treated, introduction of treadmill grade can increase metabolic demand. For every $2 \%$ elevation in treadmill incline, there is a $6.4 \%$ increase in $\mathrm{VO}_{2}$. Thus, depending on the injury type and goals for the patient, grade or speed can be adjusted to maintain fitness during the recovery period. Conversion tables have been developed for the translation of speed matching with an antigravity treadmill. ${ }^{10}$ Linear regression equations were developed for speeds between 6.4 and $16.1 \mathrm{~km} / \mathrm{h}$ (4 and $10 \mathrm{mph}$ ) in $0.8 \mathrm{~km} / \mathrm{h}(0.5 \mathrm{mph})$ increments and between the BWS of 50 and $100 \%$, in

Table 1A. Antigravity Treadmill Training Progressions for Sample Injury Cases: Osteochondral Repair in Knee (Initiated 9 months postsurgery)

\begin{tabular}{|c|c|c|c|c|}
\hline & Sessions & Speed & Percent & $\begin{array}{c}\text { Running Time Bouts: } \\
\text { Walk Bouts }\end{array}$ \\
\hline Week & Per Week & (mph) & Body Weight & $(\min )$ \\
\hline 1 & 1 & 6.7 & 30 & $5: 25$ \\
\hline 2 & 1 & 7.2 & 30 & $10: 20$ \\
\hline 3 & 2 & $7.6,7.7$ & 40,40 & 10-15: 20-15 \\
\hline 4 & 2 & $7.5,8.0$ & 50,50 & $15-20: 10-15$ \\
\hline 5 & 2 & 8.38 .0 & 60,60 & $20-25: 5-10$ \\
\hline 6 & 2 & $7.5,7.1$ & 70,70 & $25: 5$ \\
\hline 7 & 1 & 8.0 & 80 & $25: 5$ \\
\hline 8 & 1 & 7.5 & 80 & $25: 5$ \\
\hline 9 & $\begin{array}{l}\text { Begin ground } \\
\text { running }\end{array}$ & & & \\
\hline
\end{tabular}


$10 \%$ increments. These conversions can guide rehabilitation teams on developing protocols to help runners with fitness goals, while recovering from injury. Fig 2 provides a sample conversion table for comparable running speeds to maintain aerobic stimuli similar to running with full body weight.

\section{Case Studies}

Two sample case studies are presented next, with different running-related injuries, treatment courses, and tissue-healing goals. These cases were previously reported ${ }^{33}$ or developed from published reports. ${ }^{1,34}$ Sample antigravity treadmill protocols are provided.

\section{Case 1. Osteochondral Repair}

Patient: An otherwise healthy, 39-year-old endurance runner (female; $60.3 \mathrm{~kg}$, running events $10-\mathrm{K}$ marathon over last 6 years); forefoot striker and averages $41.6 \mathrm{~km} / \mathrm{wk}$ at a running pace of $7.5-8 \mathrm{~min} / \mathrm{mile}$. She presented clinically with two years of knee pain and femoral cartilage medial grade $3-4$ defect of $3 \mathrm{~cm}^{2}$ and grade 1 lateral tibial cartilage defect. ${ }^{33}$ She underwent single-step arthroscopic osteochondral repair surgery, which included microfracture and bone marrow aspirate concentrate. Postsurgery, the patient was partial weight-bearing for 2 weeks, then weighted, as tolerated, while wearing a cartilage brace with a medial hinge. Postoperative rehabilitation consisted of nonimpact exercise (swimming, cycling) and leg strengthening. Given that healing and cartilage maturation can require up to three years, performing optimal loading during the prolonged rehabilitation period is critical.

At month nine, the patient had full knee range of motion and was cleared to begin an 8-week graduated return-to-run program. Twelve sessions of antigravity

Table 1B. Antigravity Treadmill Training Progressions for Sample Injury Cases: Distal Medial Tibial Stress Reaction (Initiated 4 Weeks After Diagnosis)

\begin{tabular}{|c|c|c|c|c|}
\hline & Sessions & Speed & Percent & \\
\hline Week & $(\mathrm{mph})$ & $\begin{array}{c}\text { Body } \\
\text { Weight }\end{array}$ & $(\min )$ & $\begin{array}{l}\text { RT Bouts in } \\
\text { 30-min Session }\end{array}$ \\
\hline$\overline{1}$ & 2 & $5-7$ & 50 & 1 min $4-5$ times \\
\hline 2 & $2-3$ & $5-7$ & 60 & $1-2$ min 5 times \\
\hline 3 & $2-3$ & $5-7$ & 70 & $1-3$ min 5 times \\
\hline 4 & $2-3$ & $5-8$ & 80 & $2-3$ min 5 times \\
\hline 5 & 3 & $5-8$ & 90 & 3-4 min 5 times \\
\hline 6 & 3 & $5-8$ & 100 & 4-5 min 5 times \\
\hline 7 & Begin ground running & & & \\
\hline
\end{tabular}

treadmill training were performed, with each starting and ending with self-paced walking at $100 \%$ body weight for 5 minutes (Table 1A). No pain was reported by the patient during any treadmill session. Self-efficacy increased by 57\% from week 1 to week 8 . This finding is clinically relevant, and better self-efficacy is related to better functional and rehabilitation outcomes after knee injury and less fear of returning to running. ${ }^{35}$ Thus, antigravity treadmill training facilitated the safe return to running, while promoting a positive psychological outlook on running capability. ${ }^{33}$

\section{Case 2. Distal Medial Tibial Stress Fracture}

Patient: A 24-year old elite competitive runner developed distal medial tibial pain. He had experienced pain in the same location 6 months earlier, which resolved with 3 weeks of rest. During the clinical exam, he reported progressively worsening pain to the point where it hurt with walking and running. He had a positive "hop" test and pain upon palpation. He was placed in a walking boot, and magnetic resonance imaging confirmed suspicion of a stress fracture in the medial tibia. The runner was prescribed 3 weeks of rest with boot and was reexamined for pain. Core and truncal stabilization and strengthening exercises were initiated immediately. At week 3, the pain had disappeared with walking, and he began walking on the antigravity treadmill at 4 weeks postdiagnosis (see Table 1B).

The program was designed to introduce gradual loading with very short jogging intervals interspersed with brisk walking. The total time during each treadmill session was 30 minutes, and a progressive increase in running bout time was introduced in BWS until week 6 . The progression in speed and reloading was dependent on symptoms; during the program, the runner did not experience any pain at the injury site and was able to run without a limp. By week 7, the runner began short bouts of outdoor running and experienced a gradual return to preinjury distance without resumption of pain. It is important to recognize that pain will be the primary indicator of whether or not the antigravity treadmill rehabilitation needs modification.

\section{Conclusions}

Antigravity treadmills can provide several therapeutic advantages for running injury rehabilitation, including preservation of aerobic fitness, muscle activation patterns and muscle mass during recovery compared to traditional rehabilitation protocols. Speed or grade can be increased to maintain metabolic demand and fitness while minimizing bone and tissue loading. Monitoring pain symptoms during antigravity treadmill training will guide protocol adjustments to BWS and prescription. When pain-free running is achieved at $>95 \%$ 


\section{BWS for $>30$ min, the runner is likely ready to safely transition to ground running.}

\section{References}

1. Liem BC, Truswell HJ, Harrast MA. Rehabilitation and return to running after lower limb stress fractures. Curr Sports Med Rep 2013;12:200-207.

2. Saxena A, Granot A. Use of an anti-gravity treadmill in the rehabilitation of the operated Achilles tendon: A pilot study. J Foot Ankle Surg 2011;50:558-561.

3. McNeill DKP, Kline JR, de Heer HD, Coast JR. Oxygen consumption of elite distance runners on an anti-gravity treadmill. J Sports Sci Med 2015;14:333-339.

4. de Heer HD, Kaufman A, Repka CP, Rojas K, Charley B, Bounds R. AlterG anti-gravity treadmill accuracy of unloading is affected by support frame height. J Strength Cond Res 2021;35:2910-2914.

5. Kim P, Lee H, Choi W, Jung S. Effect of 4 weeks of antigravity treadmill training on isokinetic muscle strength and muscle activity in adult patients with a femoral fracture: A randomized controlled trial. Int J Environ Res Public Health 2020;17:E8572.

6. Coyle EF, Martin WH, Sinacore DR, Joyner MJ, Hagberg JM, Holloszy JO. Time course of loss of adaptations after stopping prolonged intense endurance training. J Appl Physiol Respir Environ Exerc Physiol 1984;57: 1857-1864.

7. Wagner M, Dames KD. Making the grade: An exploration of incline running on a bodyweight-supportive treadmill. J Sport Rehab 2021;12:1-5.

8. Warden SJ, Davis IS, Fredericson M. Management and prevention of bone stress injuries in long-distance runners. J Orthop Sports Phys Ther 2014;44:749-765.

9. Grabowski AM, Kram R. Effects of velocity and weight support on ground reaction forces and metabolic power during running. J Appl Biomech 2008;24:288-297.

10. Kline JR, Raab S, Coast JR, Bounds RG, McNeill DKP, de Heer HD. Conversion table for running on lower body positive pressure treadmills. J Strength Cond Res 2015;29: 854-862.

11. Raffalt PC, Hovgaard-Hansen L, Jensen BR. Running on a lower-body positive pressure treadmill: $\mathrm{VO}_{2} \mathrm{max}$, respiratory response, and vertical ground reaction force. Res $Q$ Exerc Sport 2013;84:213-222.

12. Gojanovic B, Cutti P, Shultz R, Matheson GO. Maximal physiological parameters during partial body-weight support treadmill testing. Med Sci Sports Exerc 2012;44: 1935-1941.

13. Stockland J, Russell Giveans M, Ames P. The effect of an anti-gravity treadmill on running cadence. Int J Sports Phys Ther 2019;14:860-865.

14. Moran MF, Rickert BJ, Greer BK. Tibial acceleration and spatiotemporal mechanics in distance runners during reduced-body-weight conditions. J Sport Rehabil 2017;26: 221-226.

15. Neal M, Fleming N, Eberman L, Games K, Vaughan J. Effect of body-weight-support running on lower-limb biomechanics. J Orthop Sports Phys Ther 2016;46:784-793.

16. Sainton P, Nicol C, Cabri J, Barthelemy-Montfort J, Berton E, Chavet P. Influence of short-term unweighing and reloading on running kinetics and muscle activity. Eur J Appl Physiol 2015;115:1135-1145.

17. Jensen BR, Hovgaard-Hansen L, Cappelen KL. Muscle activation and estimated relative joint force during running with weight support on a lower-body positivepressure treadmill. J Appl Biomech 2016;32:335-341.

18. Brennan CT, Jenkins DG, Osborne MA, Oyewale M, Kelly VG. Can anti-gravity running improve performance to the same degree as over-ground running? J Sports Sci 2018;36:2273-2281.

19. Cutuk A, Groppo ER, Quigley EJ, White KW, Pedowitz RA, Hargens AR. Ambulation in simulated fractional gravity using lower body positive pressure: Cardiovascular safety and gait analyses. J Appl Physiol (1985) 2006;101:771-777.

20. Masumoto K, Soucy MT, Bailey JP, Mercer JA. Muscle activity during backward and forward running with body weight support. Hum Mov Sci 2017;55:276-286.

21. Debecker N, Luyten M, Vandenabeele F, Bellemans J. The effect of anti-gravity training after meniscal or chondral injury in the knee. A systematic review. Acta Orthop Belg 2020;86:422-433.

22. Patil S, Steklov N, Bugbee WD, Goldberg T, Colwell CW, D'Lima DD. Anti-gravity treadmills are effective in reducing knee forces. J Orthop Res 2013;31:672-679.

23. Smoliga JM, Wirfel LA, Paul D, Doarnberger M, Ford KR. Effects of unweighting and speed on in-shoe regional loading during running on a lower body positive pressure treadmill. J Biomech 2015;48:1950-1956.

24. Hansen C, Einarson E, Thomson A, Whiteley R, Witvrouw E. Hamstring and calf muscle activation as a function of bodyweight support during treadmill running in ACL reconstructed athletes. Gait Posture 2017;58: 154- 158.

25. Liebenberg J, Scharf J, Forrest D, Dufek JS, Masumoto K, Mercer JA. Determination of muscle activity during running at reduced body weight. J Sports Sci 2011;29: 207-214.

26. Henkelmann R, Palke L, Schneider S, et al. Impact of antigravity treadmill rehabilitation therapy on the clinical outcomes after fixation of lower limb fractures: A randomized clinical trial. Clin Rehabil 2021;35:356-366.

27. Tenforde AS, Watanabe LM, Moreno TJ, Fredericson M. Use of an antigravity treadmill for rehabilitation of a pelvic stress injury. PM R 2012;4:629-631.

28. Moore MN, Vandenakker-Albanese C, Hoffman MD. Use of partial body-weight support for aggressive return to running after lumbar disk herniation: A case report. Arch Phys Med Rehabil 2010;91:803-805.

29. Hoffman MD, Donaghe HE. Physiological responses to body weight-Supported treadmill exercise in healthy adults. Arch Phys Med Rehabil 2011;92:960-966.

30. Ebert JR, Ackland TR, Lloyd DG, Wood DJ. Accuracy of partial weight bearing after autologous chondrocyte implantation. Arch Phys Med Rehabil 2008;89: 1528-1534.

31. Farina KA, Wright AA, Ford KR, Wirfel LA, Smoliga JM. Physiological and biomechanical responses to running on lower body positive pressure treadmills in healthy populations. Sports Med 2017;47:261-275.

32. Brüssau T, Oehring R, Felix SB, Dörr $M$, Bahls $M$. Cardiorespiratory and metabolic responses to exercise 
testing during lower-body positive pressure running. J Appl Physiol (1985) 2020;128:778-784.

33. Hambly K, Poomsalood S, Mundy E. Return to running following knee osteochondral repair using an anti-gravity treadmill: A case report. Phys Ther Sport 2017;26:35-40.

34. Saxena A, Fullem B, Gerdesmeyer L. Treatment of medial tibial stress syndrome with radial soundwave therapy in elite athletes: current evidence, report on two cases, and proposed treatment regimen. J Foot Ankle Surg 2017;56: 985-989.

35. Chmielewski TL, Zeppieri G, Lentz TA, et al. Longitudinal changes in psychosocial factors and their association with knee pain and function after anterior cruciate ligament reconstruction. Phys Ther 2011;91:1355-1366. 